\title{
High-Speed Simulation of the GPRS Link Layer
}

\author{
J Gozalvez and J Dunlop \\ Department of Electronic and Electrical Engineering, University of Strathclyde \\ 204 George St, Glasgow G1-1XW, Scotland \\ Tel: +44141548 2061, Fax: +441415524968 \\ j.gozalvez@comms.eee.strath.ac.uk, j.dunlop@eee.strath.ac.uk
}

\begin{abstract}
The General Packet Radio Service (GPRS) is due to be implemented in current mobile networks, introducing a whole new range of mobile data applications and services. Considerable effort has been devoted to test its capabilities and system performance. In this paper, a modular and high-speed GPRS simulator is presented. In order to validate the simulation method, the GPRS link layer performance is assessed. The performance, for a single slot packet data channel, is evaluated in a typical urban environment for a range of mobile velocities. The impact of frequency hopping on link quality measures is also evaluated.
\end{abstract}

\section{INTRODUCTION}

The General Packet Radio Service (GPRS) [1] has been developed as a standardised system for the provision of packet data services for both evolved GSM and TDMA/136 networks. Its higher bandwidth efficiency compared to circuit switched systems, such as GSM, is due to the introduction of "capacity on demand" and the statistical multiplexing of users in a single slot. GPRS also provides increased data rates through the allocation of multiple slots to a single user, reaching a maximum throughput of $171.2 \mathrm{kbits} / \mathrm{s}$ when considering eight slots per user.

GPRS introduces three new channel coding schemes. The fourth GPRS coding scheme, the more robust one, is already used by GSM for the Slow Associated Control Channel. These coding schemes provide different degrees of error protection. Their impact on the radio link quality needs then to be assessed. For this purpose, an enhanced software version of a simulator previously used to evaluate the impact of Link Adaptation in GSM [2], has been used. By means of this high-speed simulator, the GPRS radio link performance in a typical urban environment, under different operating conditions, is evaluated. The work has considered the allocation of a single slot per frame. The outcome of this work not only represents an independent verification of the GPRS link layer performance, but also an assessment of the capabilities of the simulator for the study of packet based systems such as GPRS or EDGE.

This paper is organised as follows. In section II, the characteristics of the GPRS channel coding schemes are described. The simulator and method used for this work are presented in section III. The GPRS link layer results are shown and discussed in section IV. Finally, conclusions are drawn, and some possibilities of future research are evoked.

\section{GPRS CHANNEL CODING}

Prior to transmission, GPRS data packets are segmented into smaller data blocks. The segmentation process is depicted in figure 1.

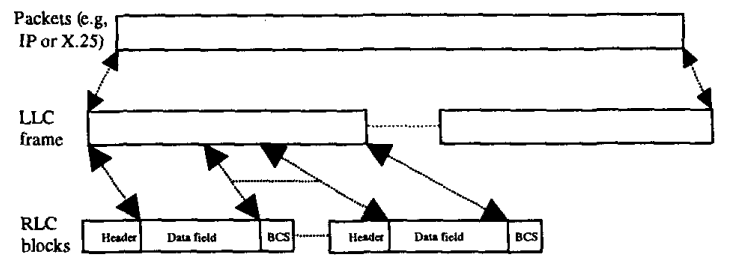

Figure 1 Packet transmission flow

The data packets, received from network layers, are first split into Logical Link Control (LLC) frames. The LLC frames are then segmented into Radio Link Control (RLC) blocks. The resulting RLC data blocks are then coded and block-interleaved over four normal bursts in consecutive TDMA frames. The RLC block's data field length will depend on the channel coding schemes used.

Four channel coding schemes, CS1 to CS4, are specified for the GPRS packet data traffic channels [3]. Each scheme has been designed to provide different resilience to propagation errors under unfavourable radio conditions, as depicted in figure 2 .

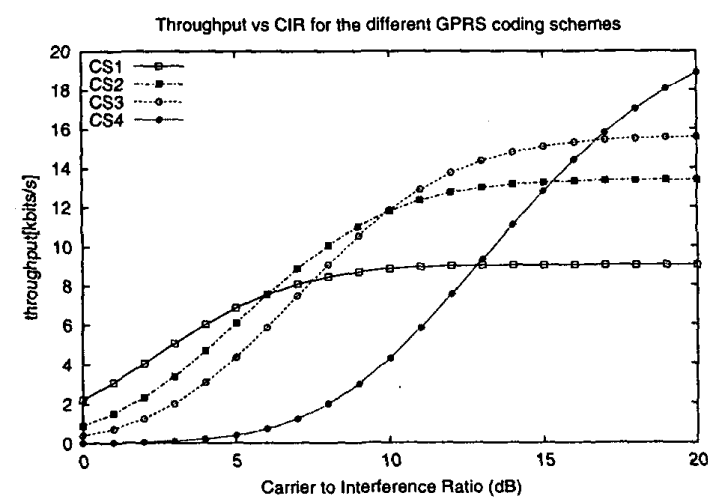

Figure 2 Performance of the GPRS coding schemes

$0-7803-6465-5 / 00 \$ 10.00 @ 2000$ IEEE 
The different coding schemes offer a trade-off between throughput and coding protection, paving the way for the application of Link Adaptation to GPRS. Coding rates of $1 / 2,2 / 3,3 / 4$ and 1 are provided. CS 1 corresponds to the more robust scheme while CS4 does not use any error correction. CS1 to CS3 are based on a half rate convolutional encoder. However, they differ on the puncturing schemes applied to the output of this encoder. Block Check Sequences are used in all the schemes to facilitate the error detection at the receiver. The outcome of the coding process is a block of 456 bits. The encoding process is illustrated in figure 3 while the characteristics of the different coding schemes are summarised in table 1 .

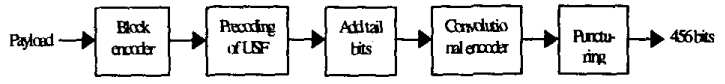

Figure 3 GPRS coding process

Table 1 GPRS channel coding parameters

\begin{tabular}{|c|c|c|c|c|c|c|c|c|c|}
\hline $\begin{array}{c}\text { Sche } \\
\text { me }\end{array}$ & $\begin{array}{c}\text { Cod } \\
\text { rate } \\
\text { rate }\end{array}$ & USF & $\begin{array}{c}\text { Pre- } \\
\text { code } \\
d \\
\text { USF }\end{array}$ & $\begin{array}{c}\text { Radio } \\
\text { Block } \\
\text { excl. } \\
\text { USF } \\
\text { and } \\
\text { BCS }\end{array}$ & $\begin{array}{c}\text { BCS } \\
\text { (parity } \\
\text { bits) }\end{array}$ & Tail & $\begin{array}{c}\text { Coded } \\
\text { bits }\end{array}$ & $\begin{array}{c}\text { Pun } \\
\text { ctur } \\
\text { od } \\
\text { bits }\end{array}$ & $\begin{array}{c}\text { Data } \\
\text { rate } \\
k b / s\end{array}$ \\
\hline CS-1 & $1 / 2$ & 3 & 3 & 181 & 40 & 4 & 456 & 0 & 9.05 \\
\hline$C S-2$ & $\approx 2 / 3$ & 3 & 6 & 268 & 16 & 4 & 588 & 132 & 13.4 \\
\hline$C S-3$ & $\approx 3 / 4$ & 3 & 6 & 312 & 16 & 4 & 676 & 220 & 15.6 \\
\hline$C S-4$ & 1 & 3 & 12 & 428 & 16 & 0 & 456 & 0 & 21.4 \\
\hline
\end{tabular}

A common characteristic of all the coding schemes is the presence of the Uplink State Flag (USF) in the RLC blocks header. The USF is transmitted in the downlink to indicate which Mobile Station (MS) should transmit in the next uplink slot. The USF comprises three bits at the beginning of each downlink radio block. It enables the coding of eight different USF states used to multiplex different MSs. Given its importance, a precoding process is applied to the three bits representing the USF; as shown in table 1.

\section{SIMULATION METHOD}

This section describes the simulation method used for this work. The significant reduction in simulation time is emphasised.

\section{A. Testbed}

An enhanced software version of the demonstrator reported in [2] has been used in order to study the performance of the GPRS Link Layer. This simulator models the transmission chain through the use of a database of error patterns produced with the bit level simulation package COSSAP [5]. Figure 4 illustrates the GPRS transmission chain. Co-channel interference has been modelled as a single continuous strong interferer, following the characteristics of the testbed used by ETSI [4]. The thermal noise at the receiver has also been included.

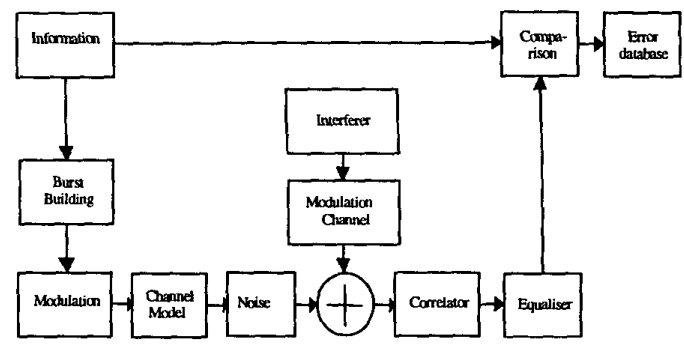

Figure 4 Transmission chain

The GPRS channel coding/decoding functions use the error database as illustrated in figure 5. When simulating the physical layer, the channel coding output is first interleaved and then the radio propagation effects are added. The output of this sum is then de-interleaved before being passed to the channel decoding process. However, de-interleaving the error patterns and adding them to the channel coding output is equivalent. This last solution has been adopted for this simulator for the sake of simplicity.

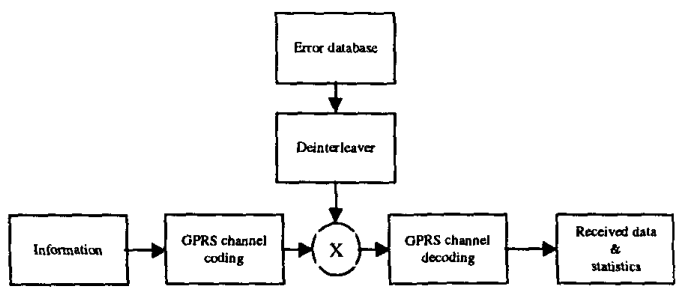

Figure 5 GPRS simulator

The derivation of an error database significantly reduces the simulation time whilst maintaining accuracy of radio link quality representation. In fact, the error database is independent of the data bits transmitted [5] making it possible to be reused whenever the radio path effects have to be taken into account.

The production of the error database (representing approximately the transmission of 150000 RLC blocks) takes around 15 days of COSSAP simulations using a Sun ULTRA Sparc 1 workstation. That is approximately the time that will be required to analyse the GPRS coding schemes performance if the transmission chain and the channel coding were simulated using COSSAP. By decoupling the simulation of the coding schemes from the transmission chain, and once the error database has been produced, the same study only needs around 11 hours with a $450 \mathrm{MHz}$ PC (with 512 Mbytes of RAM). This results in a significant reduction of $97 \%$ in simulation time. The effect of any channel coding and interleaving schemes can therefore be quickly studied. Also, due to the design of the simulator, other frequency 
hopping patterns can be simulated without the need to simulate again the transmission chain.

\section{B. Independent replications method}

A simulation run represents a particular realisation of the environment being simulated. Different simulation runs can then differ significantly from the true response of the system. In order to reduce the variance between the output of different simulation runs, a number of simulation runs must be made before making any conclusion about the reliability of the information extracted. The method selected for the simulations presented in this paper is the Independent replications method [6]. The independence between replications is introduced in the production of the error database. Seven independent replications were done, each one simulating the transmission of 21000 RLC blocks. The results presented in the following section represent the mean values from the seven independent replications with a 95\% confidence interval. To illustrate the statistical accuracy of the results, the estimated relative error [6], defined as the ratio of the half-length of the confidence interval to the sample mean, will be specified.

\section{LINK LEVEL SIMULATION RESULTS}

In order to validate the simulation approach previously described, the GPRS link layer performance is estimated in terms of the Block Error Rate (BLER) and Uplink State Flag (USF) Error Rate. The performance is assessed for the four GPRS coding schemes, CS1 to CS4. The statistical accuracy of the results presented in this section is also specified.

\section{A. Assumptions}

A single slot per frame allocation policy has been assumed for this study. The simulations presented in this paper were conducted for a typical urban channel model, as defined in [4], at two particular speeds, $5 \mathrm{~km} / \mathrm{h}$ and 50 $\mathrm{km} / \mathrm{h}$, corresponding to a pedestrian and a car scenario. The capacity limiting factor for a cellular system in a highly populated area, such as an urban environment, is the co-channel interference. Therefore only simulations for an interference-limited case are considered in this paper, hence yielding the performance versus Carrier to Interference Ratio $(\mathrm{C} / \mathrm{I})$. For the purpose of this work, the carrier frequency was set to $900 \mathrm{MHz}$.

Since GPRS has been considered for the evolution of GSM and TDMA/136, simulations were conducted with and without frequency hopping. An ideal frequency hopping scheme (hopping between four frequencies), where perfect decorrelation between bursts is assumed [4], has been simulated. As proposed in [4], the interference and the wanted signals have the same frequency hopping sequence.

\section{B. Block Error Rate}

The BLER gives an estimate of the radio link quality. It is defined as the ratio of radio blocks received in error (i.e., at least one bit error detected by the Block Check Sequence during the channel decoding) to the total number of radio blocks.

Simulation results for both speeds and without frequency hopping, are shown in figures 6 and 7. The corresponding results when implementing frequency hopping are depicted in figures 8 and 9. The BLER reference performance for packet data channels $(10 \%)$, established in [4], is also plotted in the figures.

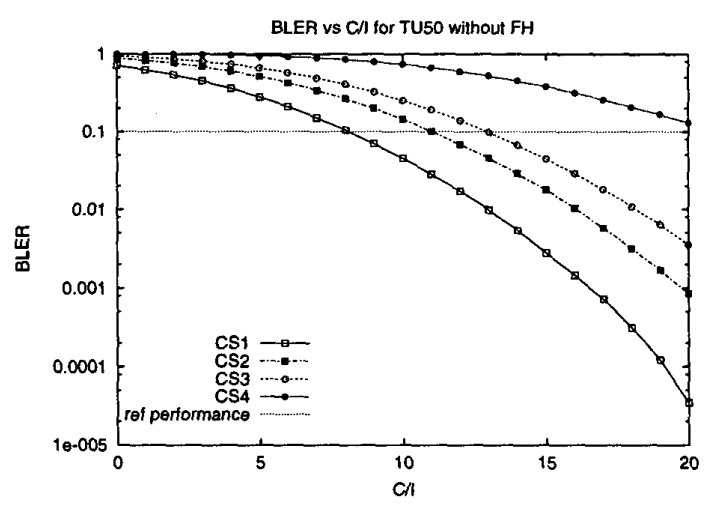

Figure 6 BLER vs C/I, without FH at $50 \mathrm{~km} / \mathrm{h}$

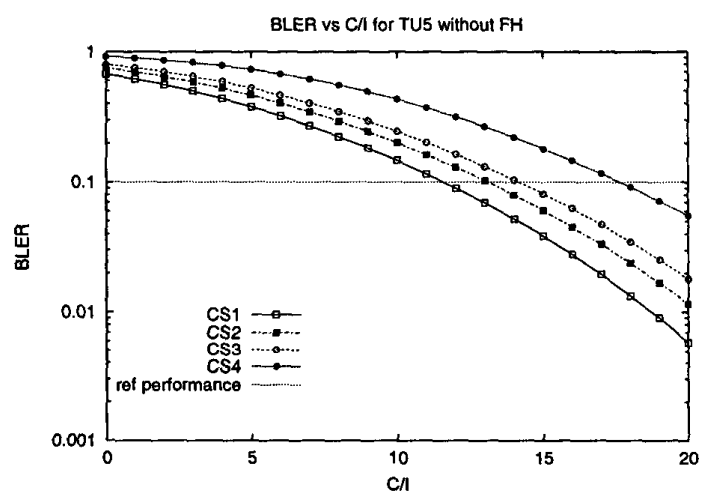

Figure 7 BLER vs C/I, without FH at $5 \mathrm{~km} / \mathrm{h}$

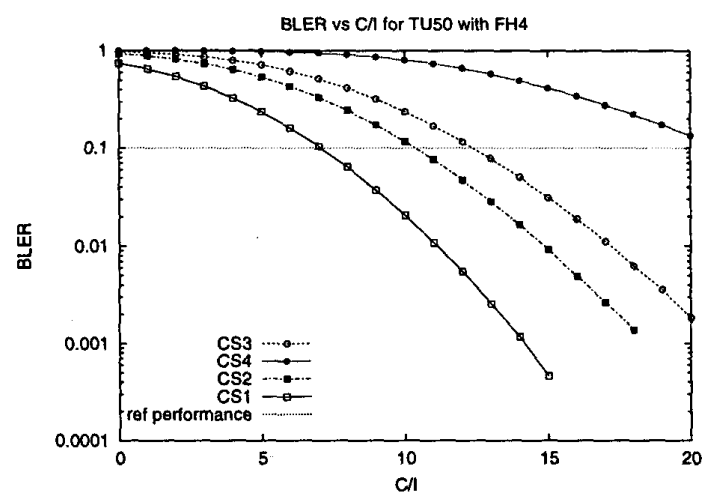

Figure 8 BLER vs C/I, with FH at $50 \mathrm{~km} / \mathrm{h}$ 


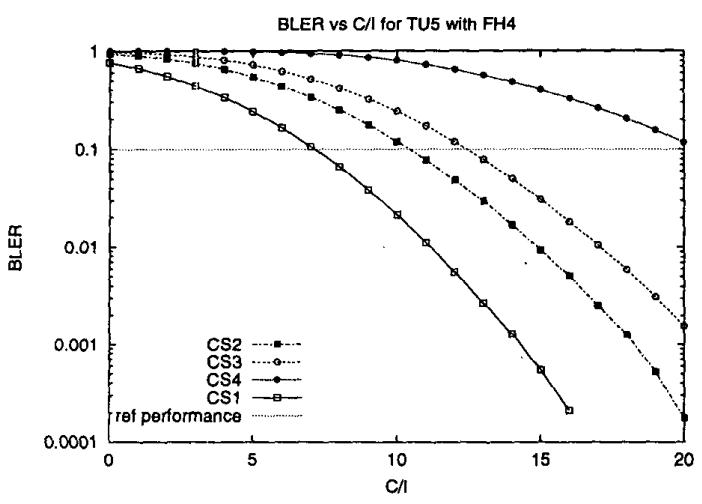

Figure 9 BLER vs C/I, with FH at $5 \mathrm{~km} / \mathrm{h}$

Figures 6 to 9 demonstrate the different robustness of the four coding schemes for varying radio conditions. These results are in accordance with those presented in [7]. By extracting the $\mathrm{C} / \mathrm{I}$ at which the $10 \%$ reference performance is reached, and comparing them with the minimum requirements imposed by ETSI [4], it may be concluded that the GPRS simulator respects ETSI standards. Figure 10 illustrates the impact of speed and frequency hopping in the BLER performance (in this case CS1).

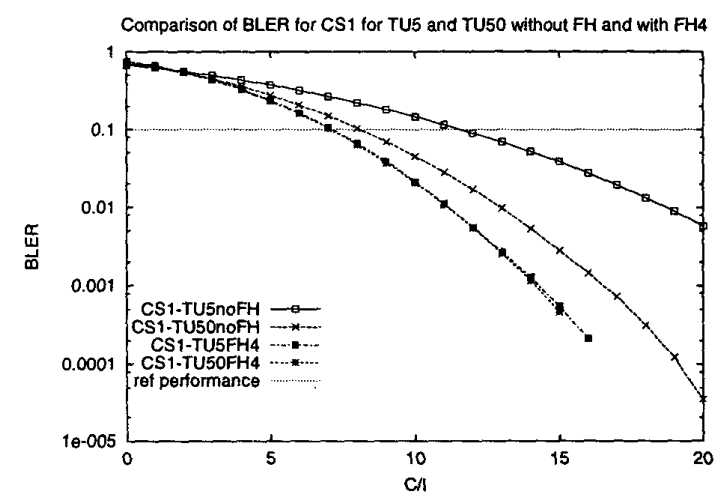

Figure 10 Impact of speed and frequency hopping on the CS1 BLER performance

The performance is significantly improved by the use of frequency hopping, independently from the mobile speed. This improvement is due to the randomisation (or spread), when using frequency hopping, of the propagation errors. With a robust scheme, spreading the errors has a positive effect as even if more blocks are affected, the length of errors is smaller and the coding scheme can correct them. The same effect is also obtained by an increase in the speed when frequency hopping is not used, but the gain in performance is lower. These characteristics are valid for CS1, CS2 and CS3 but not for CS4 as depicted in figure 11. CS4 corresponds to a particular case as a speed of $5 \mathrm{~km} / \mathrm{h}$ without frequency hopping gives the best performance. This is explained by the fact that CS4 has no coding. Spreading the errors (either by frequency hopping or high speeds) will decrease the performance since errors will affect more radio blocks, and the scheme will not be able to correct them.

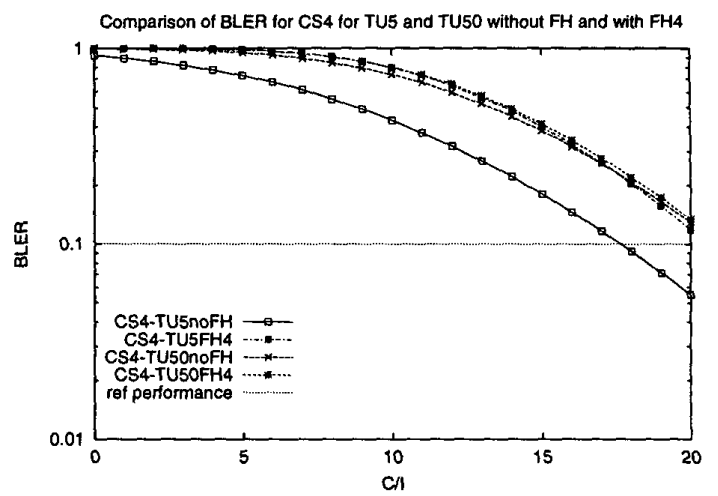

Figure 11 Impact of speed and frequency hopping on the CS4 BLER performance

The estimated relative error of the presented mean BLER values is below $5 \%$ for $\mathrm{C} / \mathrm{Is}$ in the range of 14 to $17 \mathrm{~dB}$ for all the operating conditions. The use of the CS1 coding scheme, frequency hopping and high speeds reduces the precision of the results. This is explained by the fact that the precision is dependent on the number of error events, and using CS1 or procedures that spread the propagation errors decrease the amount of errors and therefore the precision.

\section{USF Error Rate}

As previously stated, the USF Error Rate influences the Medium Access Control layer performance. It is defined as the ratio of incorrectly interpreted USFs to the total number of received USFs. The output of the USF precoding process is identical for CS2, CS3 and CS4 so only two curves will be presented in the following figures. Simulation results for both speeds and without frequency hopping are shown in figures 12 and 13. The corresponding results when implementing frequency hopping are depicted in figure 14. Due to the importance of the USF, ETSI proposes a $1 \%$ reference performance for the USF Error Rate.

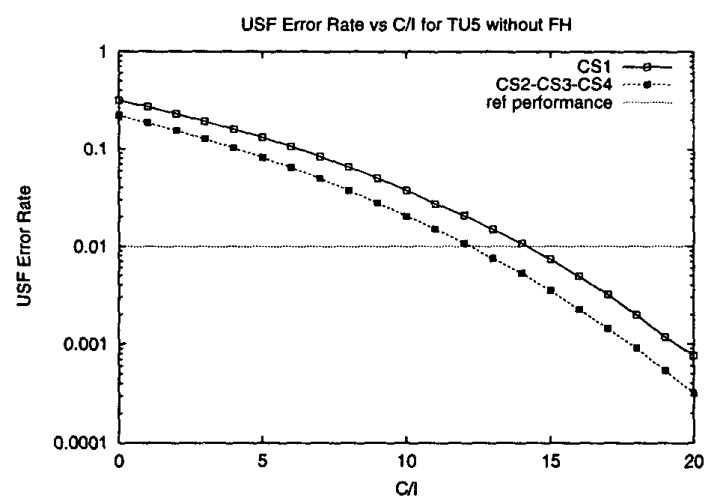

Figure 12 USF Error Rate without FH, at $5 \mathrm{~km} / \mathrm{h}$ 


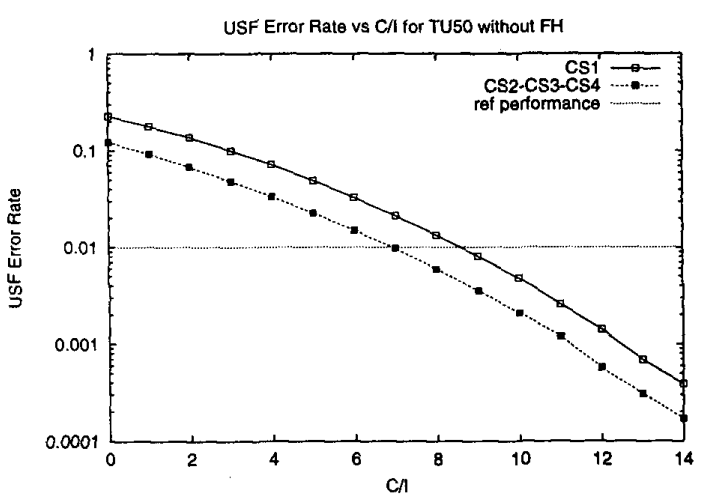

Figure 13 USF Error Rate without FH, at $50 \mathrm{~km} / \mathrm{h}$

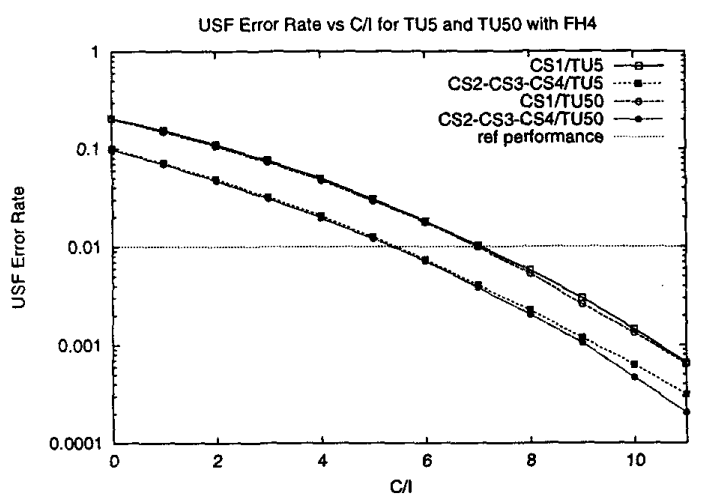

Figure 14 USF Error Rate, with FH, at $5 \mathrm{~km} / \mathrm{h}$ and $50 \mathrm{~km} / \mathrm{h}$

The CS2/CS3/CS4 USF coding shows better robustness under all operating conditions. The USF Error Rate results also respect the minimum interference ratio for which the $1 \%$ reference performance for co-channel interference shall be met [4]. A better view of the impact of the velocity and frequency hopping is depicted in figure 15. The same conclusions as for the BLER results can be drawn.

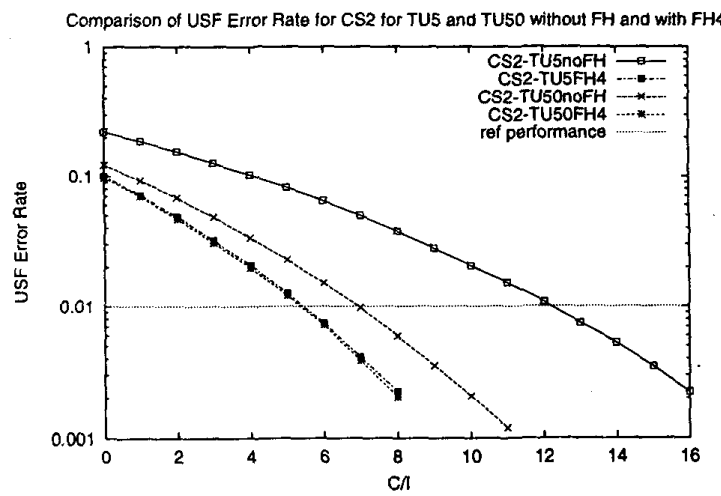

Figure 15 Impact of speed and frequency hopping on the CS2 USF Error Rate performance
The estimated relative error of the presented USF Error Rate mean values is below $10 \%$ for $\mathrm{C} / \mathrm{Is}$ of around $9 \mathrm{~dB}$ for all the operating conditions. The estimated relative error constraint was relaxed because of the strong coding schemes applied to the USF.

\section{CONCLUSIONS AND FUTURE RESEARCH}

In this paper, a high-speed simulator using an error database has been used to estimate the GPRS link layer performance by means of two link quality measures. The impact of the speed and frequency hopping on the GPRS performance has also been presented. The results show the different robustness of the GPRS channel coding schemes for varying radio conditions. High speeds and the use of frequency hopping increase the performance, except for CS4 due to its lack of error protection. The results presented in this paper are in accordance with ETSI requirements and with results presented in other papers, validating then the simulation method used.

The present study has assumed the allocation of a single slot per frame. Further research will be to assess the GPRS link layer performance when allocating several slots per frame, taking into consideration the impact of correlation between contiguous slots. Also, as GMSK modulation has been adopted as a fall back mode in EDGE [8], the error database will also be reused for future evolved versions of the simulator including EDGE.

\section{REFERENCES}

[1] ETSI, "GSM 03.64; Overall description of the GPRS radio interface. Stage 2", version 6.1.0, Release 1997.

[2] J Dunlop, J Pons, J Gozalvez, and P. Atherton, "A Real-time GSM Link Adaptation Hardware Demonstrator", to be presented at IEEE VTC2000Spring, May 15-18 2000, Tokyo.

[3] ETSI, "GSM 05.03; Channel coding", version 7.0.1, Release 98

[4] ETSI, "GSM 05.05; Radio Transmission and reception", version 7.1.0, Release 1998.

[5] J Pons, "Application of Link Adaptation to evolved TDMA mobile communications systems". PhD thesis. October 1999. University of Strathclyde.

[6] "Simulation modelling \& analysis". Averill M.Law and W. David Kelton. McGraw-Hill. $2^{\text {nd }}$ edition, 1991.

[7] P. Schramm et al, "Radio Interface Performance of EDGE, a Proposal for Enhanced Data Rates in Existing Digital Cellular Systems". Proc of IEEE VTC, May 1998, Ottawa.

[8] Furuskar, Mazur, Muller and Olofsson, "EDGE: Enhanced Data Rates for GSM and TDMA/136 Evolution". IEEE Personal Communications magazine, June 1999. 